\title{
Design of equipment training simulation system based on virtual reality technology \\ QIU Lei ${ }^{1, a}$, ZHOU Xiao ${ }^{1, b}, X^{2}$ Rongyue ${ }^{1, c}, \mathrm{HE} \mathrm{Bo}^{1, \mathrm{~d}}$ \\ ${ }^{1}$ Wuhan Mechanical College, Wuhan 430075,Hubei, China \\ aemail: hnnhlxy@163.com, bemail: leopzhx@sohu.com, \\ cemail: ryxie@126.com, demail: 22565987@qq.com
}

Keywords: Virtual reality; Equipment training; Model display; Data display

\begin{abstract}
For weapon and equipment operation training, we designed an equipment simulation system based on virtual reality technology. By multi-layer model display design, the database links, data display and simulation system design, we realized the equipment simulation system's equipment operation and tool use, enriched the equipment training method, and reduced the complex weapons training costs.
\end{abstract}

\section{Introduction}

Virtual reality technology is a virtual reality environment through digitization, giving the feeling of an immersive, which has three characteristics including immersion, interaction, and imagination. Therefore, the realization of simulation based on virtual reality technology and equipment is of great significance. Not only save a large amount of manpower and material resources, solve the inadequate training equipment, but also can be used as a training presentation materials for people to learn. The three-dimensional form relative to two-dimensional, the display effect is more realistic, operation is more convenient, more impressive to the person.

\section{The overall design of the equipment training simulation system}

Equipment training simulation system needs to achieve the equipment operation and the use of tools. The design of the simulation system is mainly divided into two parts, one is the main menu functional part, realize the switch of the basic function. Second is the realization of roaming, animation, the data display and control function, these functions are embedded in the function menu, not alone.

(1) The main menu function

The system needs to achieve the following functions: equipment roaming, operation, equipment use and troubleshooting. These functions are realized by the main menu, the main functional menu's program structure as shown in figure 1. Through the switch statement realizes the switching of four functions. 


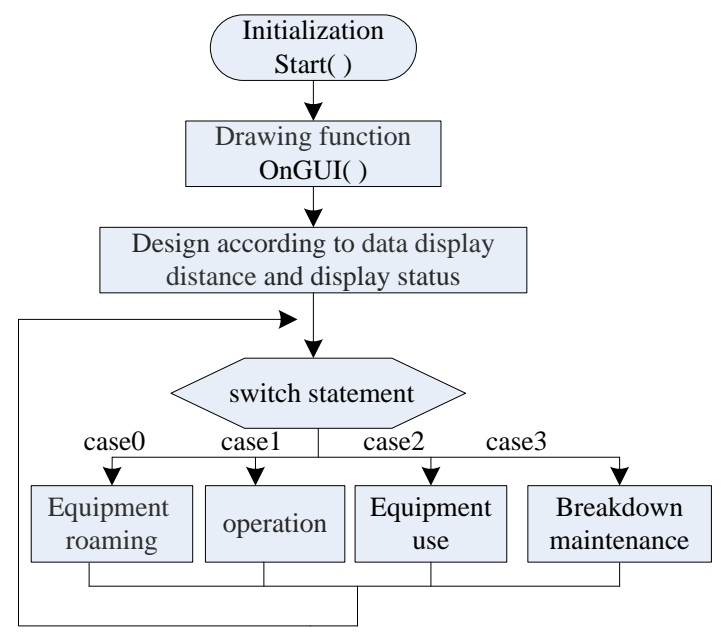

Figure.1.The functional flowchart of the main interface

(2) First-person virtual interaction

After entering the menu, the realization of first-person virtual interactions, can be gained different view of the content by adjusting the angle of view, and it also realizes equipment, equipment operation and maintenance.

(3) Data display

Through the interaction with the database, the data of virtual operation steps, time, phased tasks are displayed on the screen.

(4) Animation function

Animation function needs to achieve two kinds of animations. One is revolve of the model components, imitating the real operation. Another is to do the simulation animation, describing internal motion model and motion state.

\section{Key technology}

Equipment simulation system needs to achieve the equipment operation and the use of tools. The overall design of the simulation system is divided into the design of model design, database design and simulation system.

(1) Multi-layer model display design

In order to guarantee the fidelity of the model running effect and scene, the construction of three-dimensional model in simulation system mainly use the geometric modeling technology, the outline of objects and shapes can use point, line, polygon graphics, curve or surface equation, even images and other methods. Key parts and non-key parts of equipment respectively adopt fine modeling, fine time modeling and rough way of modeling, in the whole process of modeling, we should combine level of detail technique and the technology of texture mapping, in order to reduce the complexity of the model of real-time rendering. The display effect of monomer model equipment as shown in figure 2 .

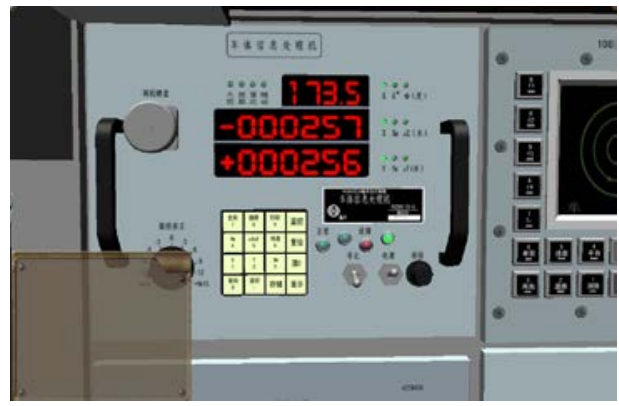

Figure.2.The display effect of monomer model

(2) Database connections

The database is the core function, mainly query data. The data need to query is divided into two kinds, one kind is the static data, such as equipment parameters, another kind is dynamic data, it 
updates once every minute. We can access static data SQL inquiry statements, dynamic data query are described below. Theoretical data should be the current time data, however, the table is the data over a period of time, so we need create a new data table to record the query time, each time the data has been read, the time nodes plus one minute. This article reads data through stored procedures and update time point, the stored procedure is as follows:

proc dushu

@shijian datetime, @value float output

as select@shijian=datatime from biaoji where id=1.0

select@value=datavalue,@shijian=datatime from ceshi where datatime= dateadd(mi,1,@shijian)

update biaoji set datatime=@shijian

After reading the data need to display through stored procedures, store it in the corresponding variable, and directly call at the time of display. The SQL statement used mainly call stored procedure, the SQL statement is as follows:

declare@shijian datetime,@value float;

execdushu@shijian,@value output select@value as datavalue

In order to prevent the database failed to read, in Unity, you can use the try catch statement.

(3) Data display

Simulation system's data display is divided into two kinds, in a fixed area display and above equipment and instrument display. The displayed data are read from the database. And they display in a fixed area. Usually the equipment information and data display at the top of the screen through the GUI.Box, display position does not move as the first Angle change.

Display above the device. The core of this display is the three-dimensional coordinates converted into two-dimensional coordinates, and then display on the screen, when the first perspective moving, the location of the equipment within the field of vision changes, corresponding data display position also changes. Above equipment shows the real-time data, it needs to read from the data constantly. The main program as shown below, npcHeight is the corrected value of Y axis, modifies the initial height of the first perspective. Name 5 stores data in the database, the data has been read from the GetData program.

void OnGUI()

\{

Vector3 worldPosition=new Vector3(transform.position.X,transform.position.y+ npcHeight,transform.position.z);

Vector3 position=camera. WorldToScreenPoint(worldPosition);

if(position.z $>0$ )

\{

//get the 2D coordinate of real objects overhead

Position=new Vector2(position.X,Screen.height-position.y);

dimian=GameObject.FindWithTag(“dimian”);

name $=$ dimian.GetComponent $<$ GetData $>($ ).name5;

Vector2 nameSize=GUI.skin.label.CalcSize(new GUIContent(name));

// set the display color of yellow

GUI.color $=$ Color.yellow;

// draw the object name

GUI.Label(new Rect(position.x-(nameSize.x/2),position.y-nameSize.y, nameSize.X,nameSize.y),name);

)

else return; 
\}

(4) Simulation system design

The 3D model will be imported into the scene, according to the position of the scene image model. In order to ensure the compact connection between the various models, no leakage and broken phenomenon, after the completion of the model's placement, we need to load the collider for the scene, to ensure role does not fall when roaming. There are two common collisions: mesh collider and box collider. Plus a mesh collider component, if the component is not completely closed, when roaming it can enter into the model, this collision is consuming system resources. Plus a box collider component, which is unable to enter the interior, however, saves system resources. In the concrete added, as the case may be, to guarantee the roaming role not drop and resource consumption to save as soon as possible. After finished loading model and Collider, we should attach script for the corresponding components one by one, check whether the script runs correctly. Simulation system effect is shown in figure 3.

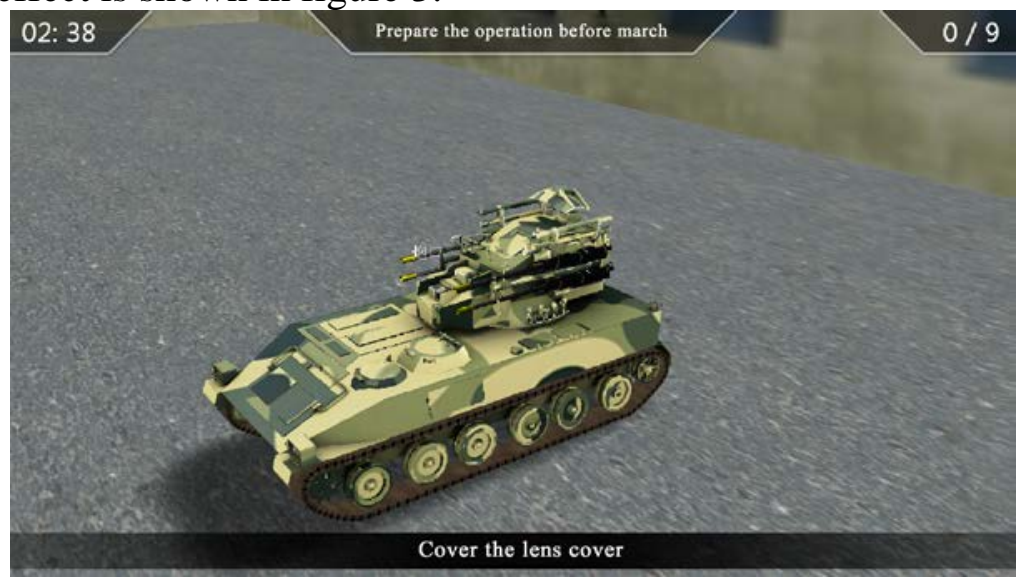

Figure.3.The effect picture of simulation system

\section{Conclusion}

The core problem of equipment training simulation system design was the model processing and data's connection. The validity in training interactive content was decided by the model's construction, and the realization of simulation richness was decided by data connection. At present, relative to practical training, simulation system had the characteristics of low cost, convenient use, and functional implementation framework was relatively simple, and it had a very good application prospect in daily training troops.

\section{References}

[1] Zhang Lili, Li Renyi. Unity3d and database communication method research [J]. Computer technology and development. 2014.

[2] Wu Yadong, Han Yongguo, Chen Bo. A 2D texture mapping method based on the triangle model [J]. Journal of computer applications and software, 2004, 21 (1): 112-114.

[3] Li Jie, Yang Na, Li Zhao. Collision detection method research in realistic game [J]. Modern computer: late on, 2014 (1) : 35-37.

[4]Li Jin, Xie Zhaoyang. SQL Server 2000 database design and system management [M]. Tsinghua University Press, 2001.

[5] Xu Lan. Research on dynamic 3D virtual scene roaming system [D]. Liaoning,; Dalian University of Technology, 2009. 